\title{
0. Why cryptoasset regulation needs rethinking: an introduction
}

\author{
Plan of the book \\ A word on terminology
}

This book has a few central concerns. How have policymakers and regulators approached the regulation of cryptoassets ${ }^{1}$ that are based on public, decentralized computer networks? Is it appropriate to apply regulations developed in the 20th century to a technology that has emerged in the 21 st century and creates new commercial and institutional possibilities? How has the approach to date impacted on the prospects for a digital ecosystem based on the underlying technology? What has influenced policy thinking and what different regulatory approach might be required, and why?

A technology that started with enabling secure, private communications has created a cascade of other possibilities. It became possible to create digital money that preserved user privacy. The technology could be used to store other things of value, such as ownership rights in property, or simply information per se. In fact, anything of value could be encoded as an exchangeable information object, leading to value and information becoming interchangeable. Private transactions using digital money to buy and sell goods and services became possible. New markets emerged, as did new market possibilities. In parallel, the oversight of state-authorized agencies was triggered, initially over national security issues. Subsequently, financial regulatory agencies became engaged as the increasing amounts of public capital committed to projects under development gave rise to concerns both familiar and novel. Are consumers at risk and are they adequately protected? What is the relationship between persons developing and/or maintaining a network and persons who access the network as users, possibly in different capacities and for different purposes? How can responsibility be allocated and accountability established in a decentralized context? How to facilitate, or not interfere with, industry development and experimentation? How to identify, manage or avoid systemic implications? Such questions become more pressing as cryptoassets increasingly interact

\footnotetext{
1 For a definition of how this term is used in this book, see "A word on terminology" at the end of this Introduction.
} 
with wider segments of society - all major jurisdictions globally have had to consider and respond to such questions since the second half of the 2010s.

The stance widely adopted by policymakers and regulators to date is that it is appropriate to apply financial regulation to cryptoassets (the standard narrative). Initially based on the need for financial capital to develop the technology, the standard narrative has had notable successes but it has not come without drawbacks. This includes uncertainties in the scope of its application, questions about whether the underlying assumptions of financial regulation have changed and its impact on the shape of industry development. While the value of the technology to various social ends and individual empowerment has been recognized, most notably by the European Parliament, ${ }^{2}$ financial regulation is unlikely to facilitate that. Thus, a theme of this book is to query the suitability and sustainability of the standard narrative and to ask whether a different approach may be required for society to reap the prospects offered by this new technology.

Multiple viewpoints are required to assess the aptness of the standard narrative and the dynamics of regulatory development to date: from the perspective of overarching statutory objectives and constraints, in light of issues created by a technology still in its early stages of evolution, and in consideration of the institutional and societal factors that influence policy thinking. In this conversation it is also important to recognize that some actors in the technology industry reject outright the notion of oversight regulation. Yet oversight regulation as a consequence of state-imposed laws is today a reality for many developers. Moreover, the governing forces of science that have always constrained and guided the development of the technology are subject in their implementation to other unavoidable considerations that the technology must confront, namely, economics and social behaviours and values more generally. At some point, interaction with the laws of society therefore seems inescapable; and inevitable to the extent the technology interacts with a heavily regulated traditional economy. The question is: where should the line be drawn, and what shape should it take with what justifications to permit, control or deny certain types of activity?

One of the difficulties in this dialogue between society and technology is to apprehend what the technology is, and what to call it. What comes together with an emerging technology is a proliferation of terms that may be used interchangeably, loosely, with embedded assumptions, or wrongly. Different persons may use the same term in different ways, sometimes for different purposes, sometimes not. This may vary across different jurisdictions and 
institutions, and for a variety of purposes including the technical, legal, regulatory, tax, accounting, functional/operational, economic or political. Semantics is important since it frequently creates an implicit discourse that confirms the positioning of the subject matter within a conceptual arena and so guides, or limits, policy thinking accordingly. The technology is rapidly evolving, and so too is the vocabulary applied to it, often resulting in core terms such as immutability and decentralization being misunderstood; this presents a variety of problems for regulators, lawmakers, and courts. ${ }^{3}$

In view of the foregoing considerations, the title of this book deserves a brief comment in view of two unorthodox terms that have been used.

The first term, cryptographic consensus technology or CCTech, has been used throughout the book. More commonly used, and popularized, terms such as blockchain and distributed ledger technology (DLT) have been avoided for two reasons. First, they are subject to frequent misuse if not misunderstanding, often bringing with them the baggage of a broader meaning that exceeds their nature. Second, and more central to an important theme of this book, neither term adequately captures nor expresses the underlying design concepts that determine the properties of any iteration of the technology, which may vary considerably. These components are referred to herein as enabling concepts, which may be configured in novel ways and, by and large, can change independently of each other. The use of the term CCTech is therefore intended to assist in refocusing attention from specific iterations of the technology to the enabling concepts that influence the characteristics of any particular iteration. Such a shift changes the conceptual level of enquiry in a manner akin to the change from studying molecules to examining the underlying atomic elements that form molecules. It gives rise to different observations and learnings. Importantly, as argued herein, such a shift has the prospect of delivering a more sustainable regulatory framework as novel ways of configuring the 'atomic elements' are found and put to new use cases. This approach reflects other efforts to gain more meaningful insights into the technology, albeit not in a regulatory context. ${ }^{4}$ The enabling concepts are introduced in Chapter 1.

3 See further: Angela Walch, 'The Path of the Blockchain Lexicon (and the Law)' (2017) 36 Rev Banking \& Fin L 713; Angela Walch, 'Blockchain's Treacherous Vocabulary: One More Challenge for Regulators' (2017) 21 J Internet L 1; Angela Walch, 'Deconstructing "Decentralization": Exploring the Core Claim of Crypto Systems' in Chris Brummer (ed), Crypto Assets: Legal and Monetary Perspectives (OUP 2019).

4 Joseph Bonneau, Andrew Miller, Jeremy Clark, Arvind Narayanan, Joshua A. Kroll, and Edward W. Felten, 'SoK: Research Perspectives and Challenges for Bitcoin and Cryptocurrencies', IEEE Symposium on Security and Privacy, 2015. 
The second term, New Prospect, is intended to cover the potential range of new possibilities that $\mathrm{CCT}$ ech creates for the formation of commercial relationships, institutional arrangements and community interactions in the context of an emerging cryptoeconomy. The New Prospect may be contrasted to the extant ways of doing things; it is to be distinguished from uses of CCTech that merely provide, for example, more efficient ways of undertaking existing commercial practices. The New Prospect is further explained in Chapter 2.

A theme running through many chapters herein is to consider how the standard narrative impacts on the ability of CCTech to deliver on the New Prospect. The view put forward is that it has changed the development of the technology in a way that has brought emphasis to financial use cases and made it more difficult for a polymorphic digital ecosystem to develop. A key proposition in this regard is that policymakers and regulatory agencies have not sufficiently interacted with the particular features of this technology and instead have sought solutions based around financial use cases and analogies to existing financial products and services. This has been supported by approaches described in later chapters of the book as incrementalist in nature, despite changed underlying assumptions that the technology presents. While the standard narrative has worked well to develop financial applications of the technology, it is not a sustainable approach to CCTech more generally, particularly as regards its institutional aspects.

Looking at CCTech through the lens of enabling concepts that may be implemented in a multitude of discovered and yet to be discovered ways, has led to the suggestion made in the final part of the book for a Determined-By-Architecture, or DBA, framework. The DBA framework focuses on the genesis of (and subsequent operation of) the nature of the connection (i.e. relationship) being established between two or more persons rather than the things exchanged (such as capital, services, rights, permissions). It introduces bottom-up considerations that may provide information more germane to achieving regulatory objectives, and can remain sustainable as a polymorphic ecosystem emerges.

The DBA framework is one of five proposals intended to provide policymakers with heuristic tools they do not currently possess to move forward from the standard narrative. As a device that may shed light on new ways of thinking about regulating $\mathrm{CCT}$ ech, the DBA framework may help develop a new starting point that is more responsive to the unique features of this technology. There is frequent talk in regulatory policy circles of the need to be technology neutral. However, as noted in the final part of the book, technology neutrality does not equate to technology agnosia. It is hoped that the DBA framework may help in understanding what it is we are trying to regulate, with what purpose and how to go about it. 


\section{PLAN OF THE BOOK}

Part I, The Initial Journey, introduces key elements of the interaction of CCTech with law and regulation. Its origins and evolution from discoveries and developments over the period from the late 1970s through to the 1990s are discussed to provide a perspective on the technology unaffected by the emergence of Bitcoin and the widespread appearance of the initial coin offering (ICO) circa 2015. It also illustrates how later regulatory responses began to position the technology leading to its current regulatory treatment.

Part II, The Primary Issues, commences with a detailed commentary on how securities laws have come to be applied to cryptoassets, and some of the areas that pose difficulty in that approach. Core concepts such as regulatory building blocks, regulatory efficiency, and the taxonomic perimeter are introduced as essential enablers for meaningful and comprehensive regulation to be possible. The last chapter discusses elements of the technology that are still in flux and which not only render the task of finding appropriate regulatory nexuses more difficult but raise questions as to the rhythm of where and how to apply or to develop regulation, and with what overarching purpose and effect.

Part III, The Secondary Market, turns to a discussion of how regulation has been applied in relation to the secondary market in cryptoassets. Despite the regulatory issues presented in the primary market, the component parts of a more complete market system are steadily being assembled around a financial construct of cryptoassets. The regulatory and developmental responses and concerns arising in relation to different centralized and decentralized cryptoexchange models are discussed, as is the regulatory treatment of intermediaries from the traditional marketplace as well as new forms of crypto-intermediation.

Part IV, Influences and Considerations, engages in a more fundamental assessment of whether the regulatory approach taken to date is sustainable. It expands on why the standard narrative has been problematic and why there are concerns that it could limit the use of the technology and the possibilities offered by the New Prospect. The relationship between the extant regulatory paradigm and the ability of a polymorphic digital ecosystem to develop based on CCTech is discussed in view of the dynamics of regulatory development to date and the precursors to ecosystem development. It is suggested that an incrementalist approach has persevered despite changed fact patterns that alter underlying assumptions of the standard narrative. As an ecosystem begins to develop, its ability to evolve successfully will depend on whether the design of a more fit-for-purpose regulation is ready, which requires policymakers to start anticipating what new regulation might look like. 
Part V, Future Directions, considers society's past experiences of responding to change in the formation of commercial relationships before revisiting the discussion of the New Prospect. Proposals for policy development are made and conclusions are drawn on whether the current approach to regulating the technology is looking at the entire picture or has adopted a blinkered view.

\section{A WORD ON TERMINOLOGY}

Given the semantic issues wrapped up in terminology (as already noted), the following definitions are adopted for clarity of meaning and strive to remain neutral by staying close to the science that enables CCTech. As such, they are broader than those generally used in current regulatory contexts, which often make implicit assumptions about the nature of a token or an asset:

- asset - anything of value capable of being legally or beneficially owned or controlled and transferred as property by a person legally able to own or control property; ${ }^{5}$

- CCTech or cryptographic consensus technology - a computer-based mechanism that uses public-key cryptography to build consensus across a network of participating stakeholders that may be more or less decentralized. While this can encompass non-public networks, the term as used herein refers only to public networks, unless otherwise indicated;

- cryptoasset - an asset comprised in a token; ${ }^{6}$

- cryptocurrency - a cryptoasset that provides no functionality other than the ability to generate a new unique key that represents ownership qua control over the digitally written entry and is designed to serve no purpose other than a peer-to-peer version of electronic cash;

\footnotetext{
5 A broad definition of property for the purposes of English law was given by the House of Lords in National Provincial Bank v Ainsworth [1965] 2 All E.R. 472. The criteria for property established by Lord Wilberforce required it to be definable and identifiable by third parties, capable of being owned by a third party, and have a degree of permanence. This has been recently affirmed by a lower court in relation to cryptoassets in AA v Persons Unknown \& Others, Re Bitcoin [2019] EWHC 3556 (Comm) (13 December 2019).

6 The use of the term cryptoasset herein is comparable, though not identical, to the definition of cryptoasset adopted by the European Commission (see its consultation document 'On an EU Framework for Markets in Crypto-assets' (December 2019) 7 (EU Consultation), and its draft regulation for 'Markets in Crypto-Assets', art 3(1)(2)).
} 
- cryptoexchange - means any facility, including trading platforms, ${ }^{7}$ that provides mechanisms for order input, order matching, clearing and settlement (i.e. a Trading Mechanism as defined in Chapter 9) but excluding facilities concerned with derivatives on cryptoassets;

- financial regulation - existing capital markets and financial services regulation;

- governance - as discussed in Chapter 6;

- interoperability - the ability to efficiently transfer and use information uniformly across organizations, systems or components, ${ }^{8}$ as expanded upon in Chapter 6;

- promoter - the person(s) ultimately responsible for procuring the deployment of a cryptoasset in the market and any accompanying white paper or similar documentation concerning the cryptoasset; ${ }^{9}$

- regulation - any system imposed by the state that regulates an activity, whether by statute, with statutory backing or via the requirements of an agency possessing administrative powers (thus excluding self-regulation);

- self-regulation - systems which regulate an activity via the formation of an organization of participating members that adopts self-imposed rules or codes of accepted market practices, whether or not membership of the organization is voluntary or required by law, and whether or not the rules or codes are subject to the oversight of a regulatory agency;

- standard narrative - the general position that it is appropriate to apply one form or another of financial regulation to cryptoassets;

- token - a digitally written instance comprising an information object maintained on a computer code implementing CCTech that is able to be controlled, via a system of one or more keys, in accordance with the set of functions the computer code provides or permits.

\footnotetext{
7 Some persons will distinguish between an "exchange" and a "trading platform". For example: using the former term to include the provision of broker/dealer services, and the latter term to refer to marketplaces bringing together different cryptoasset holders (as per the EU Consultation, pp. 16 and 18). However, that distinction is less amenable to different arrangements than the functional approach used here.

8 Berkman Institute (2005), Roadmap for open ICT ecosystems. Available at https://cyber.harvard.edu/epolicy/roadmap.pdf

9 The market commonly refers to "issuers" of cryptoassets, which is a misleading analogy to a company that issues securities. Cryptoassets are not "issued" as such but are generated. The promoter concept is statutorily recognized in securities laws in the United States Securities Act of 1933, Hong Kong's prospectus law (Companies (Winding up and Miscellaneous Provisions) Ordinance Cap. 32), and the UK's Financial Services and Markets Act 2000.
} 